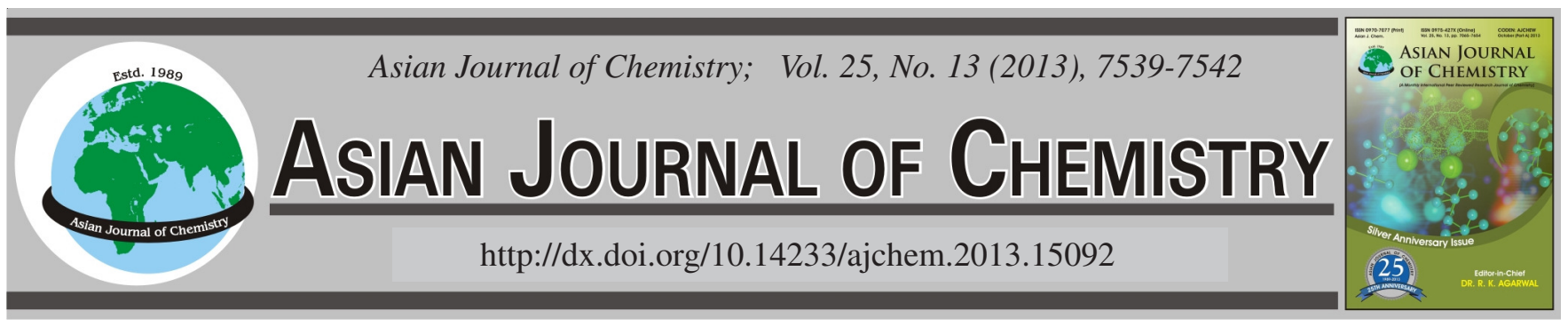

\title{
Synthesis of Fluorine Doped Zinc Oxide Particles by Hydrothermal Method
}

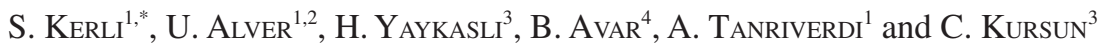

\begin{abstract}
${ }^{1}$ Department of Physics, Kahramanmaras Sutcu Imam University, 46100 Kahramanmaras, Turkey
${ }^{2}$ Department of Electrical and Electronics Engineering, International University of Sarajevo, 71210 Sarajevo, Bosnia and Herzegovina ${ }^{3}$ Research and Development Center for University-Industry and Public Relations (USKIM), 46100 Kahramanmaras, Turkey

${ }^{4}$ SNTG Lab., Physics Engineering Department, Hacettepe University, Beytepe, 06800 Ankara, Turkey
\end{abstract}

*Corresponding author: Fax: +90 344 2191042; Tel: +90 344 2191487; E-mail: suleymankerli@yahoo.com

\begin{abstract}
Fluorine doped $\mathrm{ZnO}$ particles were prepared by a simple hydrothermal technique in an autoclave at $200^{\circ} \mathrm{C}$ for $4 \mathrm{~h}$. Obtained particles were annealed at $600{ }^{\circ} \mathrm{C}$ and their physical properties were investigated. X-Ray diffraction measurements reveal that $\mathrm{ZnO}$ :F particles contain a wurtzite and simonkolleite structure. Thermal gravimetric measurements show that thermal decomposition of simonkolleite occurs at above $600{ }^{\circ} \mathrm{C}$. SEM images of particles show that the morphologies change from a flower-like structure to a rose-like structure with increasing fluorine concentration. Optical measurements revealed that absorbance edge values of fluorine doped $\mathrm{ZnO}$ particles are blue shifted.
\end{abstract}

Key Words: Fluorine doping, ZnO particles, Hydrothermal method, Zinc chloride.

\section{INTRODUCTION}

Zinc oxide with a wide band gap energy $(3.37 \mathrm{eV})$ and a hexagonal wurtzite crystal structure has attracted a great deal of attention for its potential application including piezoelectric devices, transistors, photodiodes, photo catalysis, biomedicine, etc. ${ }^{1-4}$. Various synthesis methods such as wet-chemical route ${ }^{5}$, chemical vapour deposition (CVD) ${ }^{6}$, vapour-liquid-solid $(\mathrm{VLS})^{7}$, chemical solution methods ${ }^{8}$ and hydrothermal method ${ }^{9}$ have been used to grow doped $\mathrm{ZnO}$ micro/nano-crystals. Among these methods, the hydrothermal method is promising for fabricating ideal material with special morphology because of the simple, fast, less expensive, low growth temperature, high yield and scalable process. In recent years, introducing cationic impurities in $\mathrm{ZnO}$ structure offers an effective approach to adjust structural, optical and electrical properties ${ }^{10-14}$. An alternative way to adjust the physical properties of the $\mathrm{ZnO}$ particles is the replacement of oxygen ions $\left(\mathrm{O}^{2-}\right)$ in the $\mathrm{ZnO}$ lattice by anionic impurities, such as fluorine $\left(\mathrm{F}^{-}\right)^{15}$. Most of the previous research works have focused on fluorine-doped $\mathrm{ZnO}$ thin films. There are less studies with fluorine doped $\mathrm{ZnO}$ particles. In addition, in many studies $\mathrm{ZnO}$ particles were synthesized by using zinc acetate or zinc nitrate precursor, but using zinc chloride as a precursor material is very seldom reported. In this paper, we have studied the fluorine doped $\mathrm{ZnO}$ particles (ZnO:F) prepared by hydrothermal technique using zinc chloride precursor and we have also investigated the effect of annealing on the thermal, structural, optical and morphological properties of $\mathrm{ZnO}: \mathrm{F}$ particles.

\section{EXPERIMENTAL}

The precursor solution was prepared by dissolving $0.1 \mathrm{M}$ of zinc chloride $\left(\mathrm{ZnCl}_{2}\right), 0.1 \mathrm{M}$ of hexamethylenetetramine (HMT, $\mathrm{C}_{6} \mathrm{H}_{12} \mathrm{~N}_{4}$ ) and $0.1 \mathrm{M}$ of ammonium fluoride $\left(\mathrm{NH}_{4} \mathrm{~F}\right)$ in $100 \mathrm{~mL}$ deionized water. The fluorine concentrations in precursor solutions were adjusted in terms of atomic percentage $(0,1,3$ and 5 at $\%)$ with respect to zinc. Hydrothermal growth was carried out at $200{ }^{\circ} \mathrm{C}$ in an autoclave placed on a furnace for $4 \mathrm{~h}$. After cooling naturally to room temperature, the transparent supernatant was removed by pipette and white $\mathrm{ZnO}$ precipitates were left. The obtained particles were washed with distilled water several times and dried at room temperature in air for further characterization. All experimental conditions were kept the same for all samples. To investigate the effect of annealing, the particles were annealed at $600{ }^{\circ} \mathrm{C}$ for $1 \mathrm{~h}$ in air. The crystal structures of $\mathrm{ZnO}$ particles were investigated by Philips X'Pert Pro X-ray diffractometer (XRD), with $\mathrm{CuK}_{\alpha}$ radiation, the surface morphologies were observed using a Zeiss EVO-LS10 scanning electron microscopy (SEM). Thermal gravimetric and differential thermal analysis (TG-DTA) curves were conducted with a Perkin-Elmer Diamond TG-DTA at a heating rate of $40{ }^{\circ} \mathrm{C} / \mathrm{min}$ in air. Optical properties of samples were obtained by using a Shimadzu UV-1800 ultravioletvisible spectrometer (UV-VIS). 


\section{RESULTS AND DISCUSSION}

XRD patterns of unannealed and annealed $\mathrm{ZnO}: \mathrm{F}$ particles are shown in Fig. 1a-b. Fig. 1a shows XRD patterns of $\mathrm{ZnO}: \mathrm{F}$ particles prepared in various fluorine concentration. As seen in Fig. 1a, diffraction peaks indicate that undoped $\mathrm{ZnO}$ particles have a hexagonal wurtzite structure (PDF-2, reference code: 01-079-2205). For the fluorine doped $\mathrm{ZnO}$ particles, in addition to peaks belonging to pure $\mathrm{ZnO}$ structure, some other low intensity impurity peaks have been observed at $2 \theta$ values especially between 30 and $35^{\circ}$. Moreover, the intensities of these impurity peaks increase with increasing fluorine addition, while the intensities of $\mathrm{ZnO}$ peaks are decreasing. When the fluorine concentration reaches to 5 at $\%$, a new diffraction pattern ascribed to zinc chloride hydroxide monohydrate (simonkolleite), $\mathrm{Zn}_{5}(\mathrm{OH})_{8} \mathrm{Cl}_{2} \mathrm{H}_{2} \mathrm{O}$ in accordance with the PDF2, reference code: 01-076-0922 is occurred. A detail analyses show that the impurity peaks shown in 1 and 3 at $\%$ fluorine doped $\mathrm{ZnO}$ particles arises from simonkolleite structure. The reason for the formation of this simonkolleite structure might be decrease the zinc ion concentration in precursor solution while the fluorine concentration is increasing. XRD pattern of the annealed $\mathrm{ZnO}$ nanoparticles at $600{ }^{\circ} \mathrm{C}$ for $1 \mathrm{~h}$ is shown in Fig. 1b. The absence of simonkolleite structure in the XRD data demonstrates the complete removal of impurities from $\mathrm{ZnO}: \mathrm{F}$ nanoparticle samples after annealing. In addition, as shown in Fig. 1b, the peak intensities of the planes are found to decrease with increasing fluorine doping concentration for all samples. This indicates that the crystalline quality of the $\mathrm{ZnO}: \mathrm{F}$ particles degrades when the fluorine doping increases.
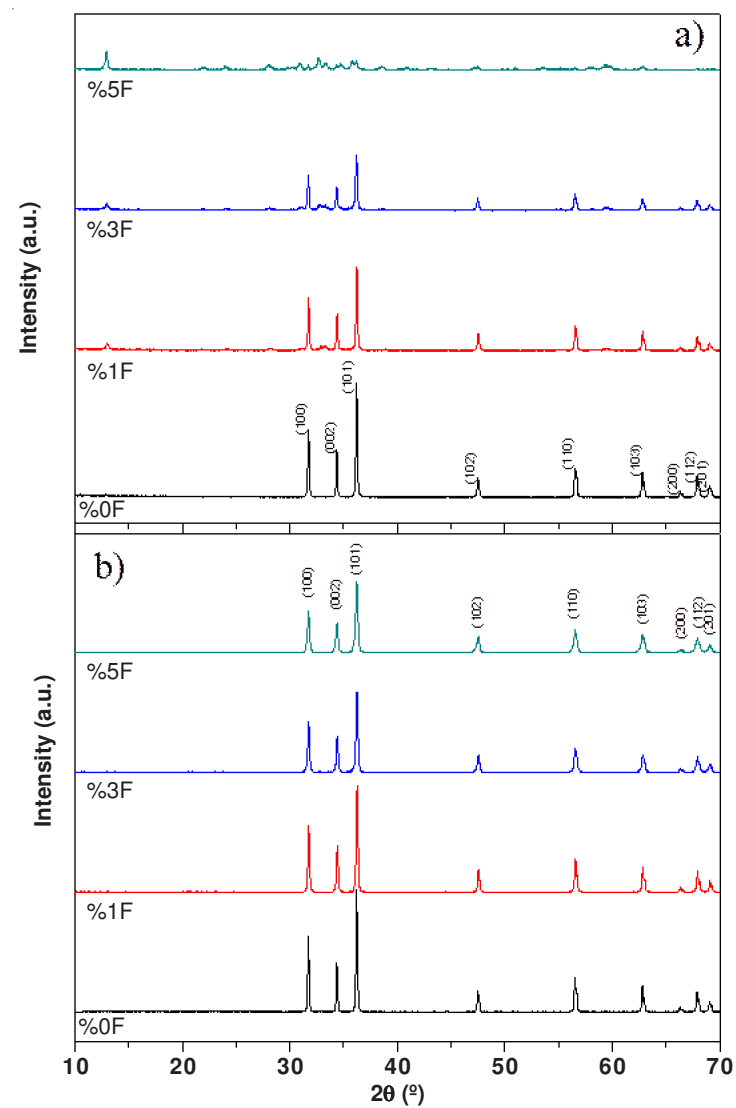

Fig. 1. (a) XRD patterns of (a) fluorine doped (b) annealed fluorine doped $\mathrm{ZnO}$ particles
TG-DTA curves of $\mathrm{ZnO}$ and 5 at. \% fluorine doped $\mathrm{ZnO}$ particles are shown Fig. 2a-b. In Fig. 2a, the TGA curves show two step weight losses. The first step at around $100-300{ }^{\circ} \mathrm{C}$ corresponds to the loss of water molecules on the surface and interlayer of the $\mathrm{ZnO}$ particles. The second step occurs at 300$600{ }^{\circ} \mathrm{C}$ due to the decomposition of hydroxide and other compounds in the precursor solutions. As seen Fig. 2a, the total weight loss is about $11 \%$ for the $\mathrm{ZnO}$ particles. For the simonkolleite structure, the first weight loss occurred at a temperature of between 100 and $300{ }^{\circ} \mathrm{C}$, which corresponded to the loss of water molecules from $\mathrm{Zn}_{5}(\mathrm{OH})_{8} \mathrm{C}_{12} \mathrm{H}_{2} \mathrm{O}$, to form $\mathrm{ZnO}$ and zinc chloride and hydrochloric acid.

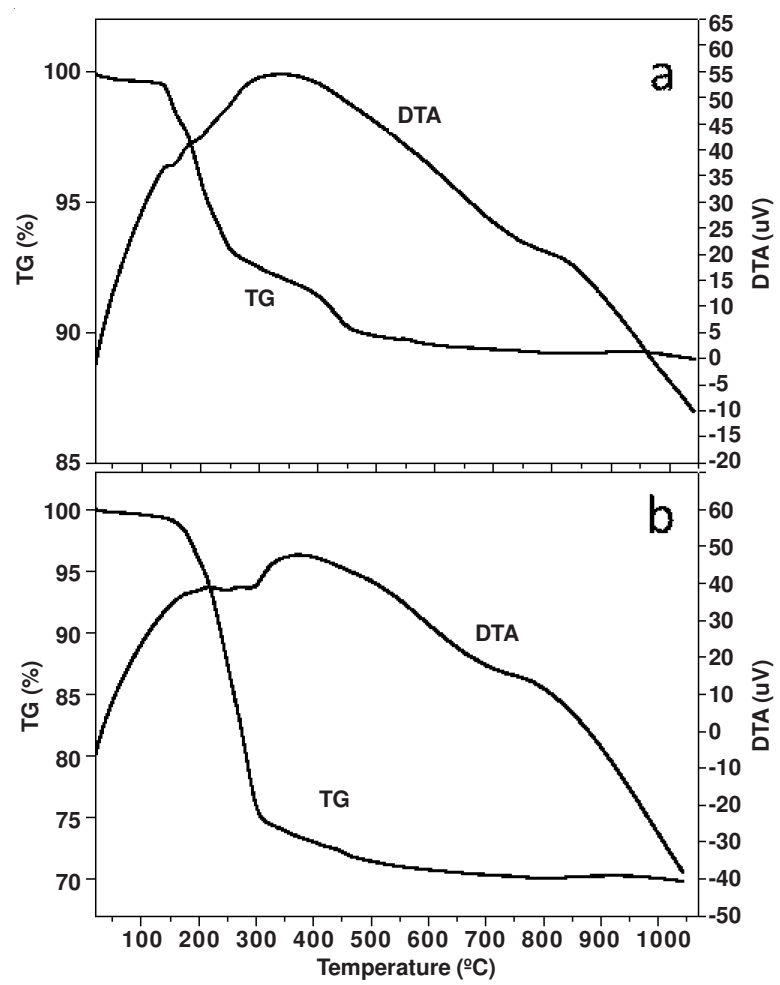

Fig. 2. TG-DTA trace for (a) $\mathrm{ZnO}$ particles (b) $5 \%$ fluorine doped $\mathrm{ZnO}$

The second weight loss occurred at in between 300 and $600{ }^{\circ} \mathrm{C}$, which was attributed to the conversion of $\mathrm{Zn}_{5}(\mathrm{OH})_{8} \mathrm{Cl}_{2} \cdot \mathrm{H}_{2} \mathrm{O}$ to complete zinc oxide. From Fig. $2 \mathrm{~b}$, the weight loss of simonkolleite structure is measured about $28 \%$ which is close to theoretical value $(26.3 \%)$. We could not observe any strong DTA peak corresponding to phase transition in Fig. 2a-b. This might be high heating rate $\left(40^{\circ} \mathrm{C} / \mathrm{min}\right)$. The results of thermal analysis indicate that the samples contain some hydroxyl group and impurities. The XRD results (Fig. 1a) confirmed this idea, because some low intensity peaks other than wurtzite $\mathrm{ZnO}$ were observed in XRD analysis for unannealed samples. As seen in Fig. 2a-b, the completion temperature of thermal-decomposing is about $600{ }^{\circ} \mathrm{C}$ and thus it was chosen as the calcination temperature to prepare $\mathrm{ZnO}: \mathrm{F}$ structures.

SEM images of prepared fluorine doped $\mathrm{ZnO}$ particles shown in Fig. 3a-d. As seen in these figures, the distribution, size and microstructure of the $\mathrm{ZnO}$ powders change with the fluorine doping concentration significantly for all samples. As seen in Fig. 3a, undoped $\mathrm{ZnO}$ particles show a flower-like 

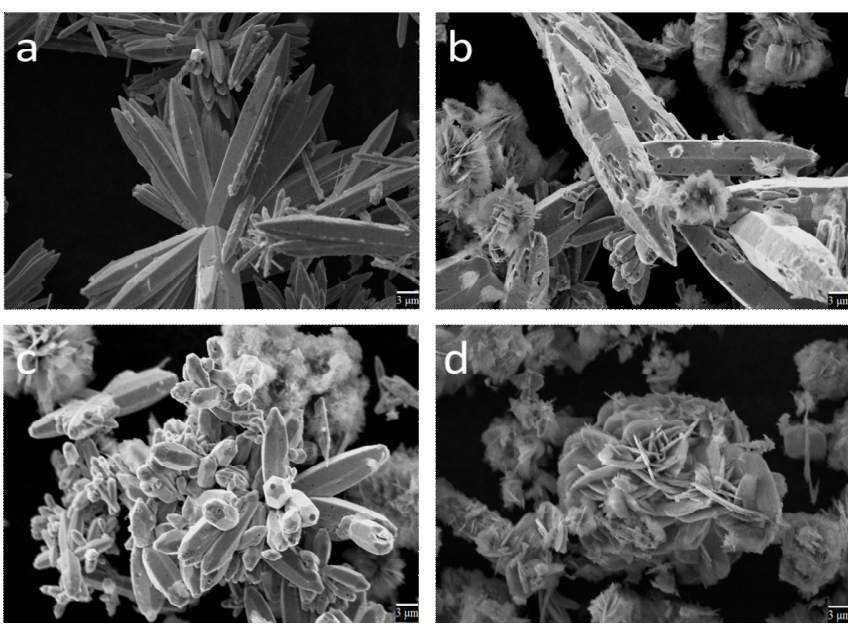

Fig. 3. SEM images of (a) $\mathrm{ZnO}$, (b) 1 at $\%$, (c) 3 at $\%$, (d) 5 at $\%$ fluorine doped $\mathrm{ZnO}$ particles

structure consisting of many sharp-tipped $\mathrm{ZnO}$ rods which originated from a single center. In Fig. $3 b$ with the fluorine adding, some rose-like structures associated with simonkolleite structure start to appear in SEM image. When the fluorine concentration was 5 at \%, the flower like structure was completely transformed to rose-like structure (Fig. 3d). These results are in agreement with the XRD result, as shown in Fig. 1a. The annealed fluorine doped $\mathrm{ZnO}$ particles are presented in Fig. 4a-d. As seen annealed images, rose-like structure assigned to simonkolleite disappears and the lengths of rod particles are decreasing with increasing fluorine concentration in structure.
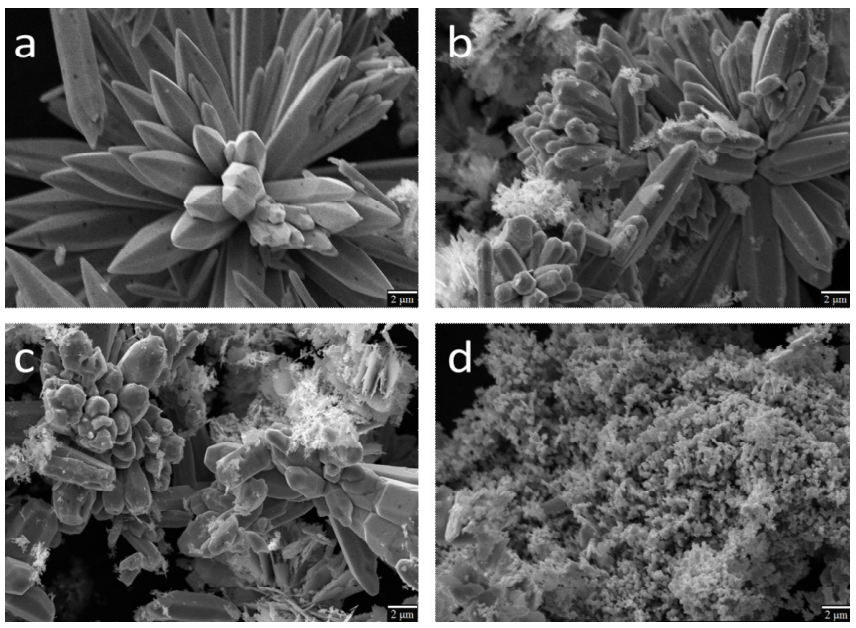

Fig. 4. SEM images of annealed (a) $\mathrm{ZnO}$, (b) 1 at $\%$, (c) 3 at $\%$, (d) 5 at $\%$ fluorine doped $\mathrm{ZnO}$ particles

The optical properties of unannealed and annealed fluorine doped $\mathrm{ZnO}$ particles were investigated by using UVvisible absorbance spectra (Fig. 5a-b). All absorption curves exhibit an observable absorption in the range 320-400 nm, with the absorption edge in between 370 and $380 \mathrm{~nm}$, due to the large exciton binding energy. Insets of Fig. 5a-b show absorbance edge shift of fluorine doped $\mathrm{ZnO}$ particles. It can be seen that the absorbance edge decreases with increasing fluorine concentration for all samples. The movement of absorbance edge to the shorter wavelength (higher band gap) is the Burnstein-Moss effect ${ }^{16}$, which is due to the increase

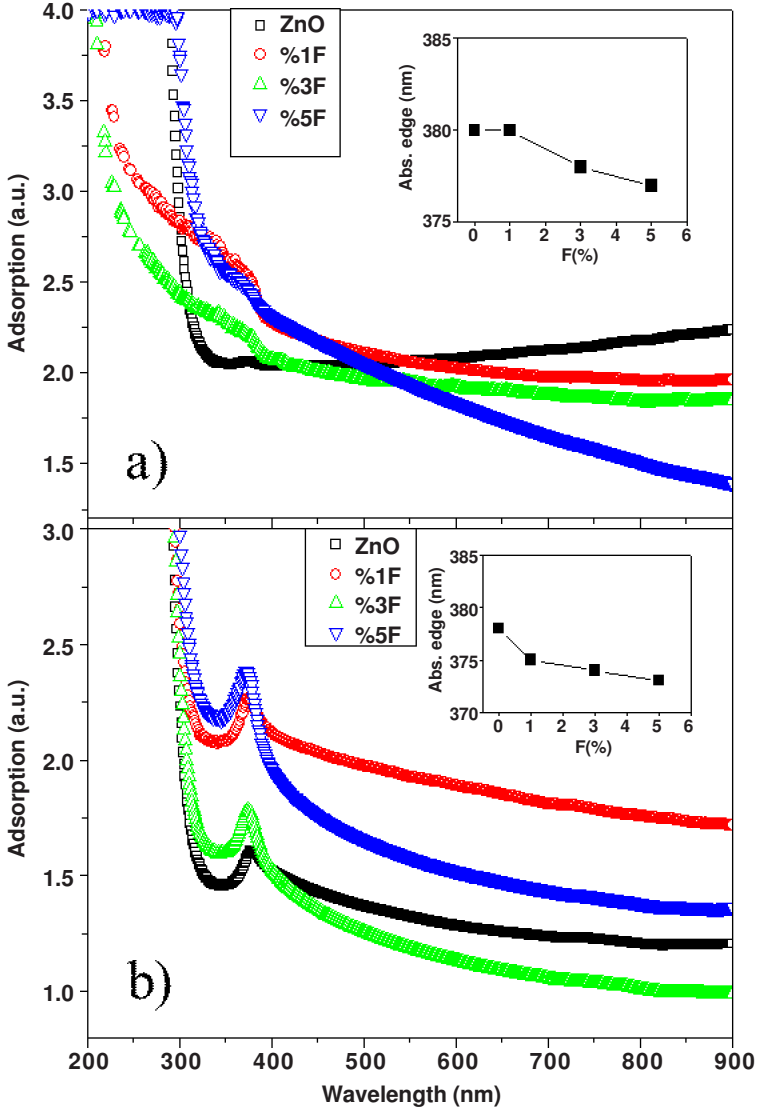

Fig. 5. Absorption spectra of (a) unannealed and (b) annealed fluorine doped $\mathrm{ZnO}$ particles. Insets show the change in absorption edge with fluorine concentration

of carrier concentration. As seen in Fig. 5b, after annealing the absorption peaks are observed much sharper and clear and the absorbance edge decreases with increasing fluorine concentration for all samples. These obtained results are in agreement with the work done by Gonzalez et al. ${ }^{8}$, they reported that the band gap energy is increased with the 5 at $\%$ fluorine doping.

\section{Conclusion}

The present investigation showed an influence of fluorine on the formation of $\mathrm{ZnO}$ particles, synthesized by hydrothermal method. XRD, SEM and TGA measurements show that obtained particles have a simonkolleite structure as well as wurtzite structure. The XRD patterns show that the wurtzite structure turns into simonkolleite structure when the doping concentration reaches to 5 at $\%$. Thermal gravimetric measurements show that thermal decomposition of simonkolleite is occurred at $600{ }^{\circ} \mathrm{C}$ and above. The morphologies of $\mathrm{ZnO}$ particles are influenced by addition of fluorine impurity. The shapes of fluorine doped $\mathrm{ZnO}$ particles are altered from a flower-like to a rose-like with increasing fluorine concentration. From UVVIS spectrum, we find that absorption edges of $\mathrm{ZnO}$ powders are decreasing with increasing fluorine concentration.

\section{REFERENCES}

1. Y.C. Kong, D.P. Yu, B. Zhang, W. Fang and S.Q. Feng, Appl. Phys. Lett., 78, 407 (2001).

2. Y.W. Zhu, H.Z. Zhang, X.C. Sun, S.Q. Feng, J. Xu, Q. Zhao, B. Xiang and R.M. Wang, Appl. Phys. Lett., 83, 144 (2003).

3. D.A. Lamb and S.J.C. Irvine, J. Cryst. Growth, 273, 111 (2004). 
4. L.A. Alkhtaby, S. Husain1, W. Khan and S.A.H. Naqvi, Asian J. Chem., 23, 5607 (2011).

5. C.-H. Ku, H.-H. Yang, G.-R. Chen and J.-J. Wu, Cryst. Growth Des., 8, 283 (2008).

6. C.L. Wu, Li Chang, H.G. Chen, C.W. Lin, T.F. Chang, Y.C. Chao and J.K. Yan, Thin Solid Films, 498, 137 (2006).

7. H. Huang, Y. Wu, H. Feick, N. Tran, E. Weber and P. Yang, Adv. Mater., 13, 113 (2001).

8. R. Gonzalez-Hernandez, A.I. Martinez, C. Falcony, A.A Lopez, M.I. Pech-Canul and H.M. Hdz-Garcia, Mater. Lett., 64, 1493 (2010).

9. R.R. Mohan, K. Rajendran, K. Sambath and O.M. Saravanakumar, Asian J. Chem., 25, S107 (2013).
10. E. Bacaksiz, S. Aksu, S. Yilmaz, M. Parlak and M. Altunbas, Thin Solid Films, 518, 4076 (2010).

11. S. Huang, Q. Xiao, H. Zhou, D. Wang and W. Jiang, J. Alloy Comp., 486, L24 (2009)

12. Y.S. Wang, P.J. Thomas and P. O'Brien, J. Phys. Chem. B, 110, 21412 (2006).

13. B.J. Lokhande, P.S. Patil and M.D. Uplane, Physica B, 302-303, 59 (2001).

14. T. Al-Harbi, J. Alloy Comp., 509, 387 (2011).

15. A. Sanchez-Juarez, A. Tiburcio-Silver and A. Ortiz, Sol. Energy Mater. Sol. Cells, 52, 301 (1998).

16. E. Burnstein, Phys. Rev., 93, 632 (1954). 